\title{
Relação entre inovação e qualidade da orientação do serviço de saúde para atenção primária
}

\author{
Cristine Hermann Nodari \\ Universidade de Caxias do Sul \\ Pelayo Munhoz Olea \\ Universidade de Caxias do Sul \\ Eric Charles Henri Dorion \\ Universidade de Caxias do Sul
}

\begin{abstract}
A inovação desempenha um diferencial na resposta às necessidades globais de saúde, pois representa a mudança que incita a evolução das organizações e conduz à maior eficiência de seus processos por meio da alocação apropriada de recursos. O artigo tem como contribuição destacar em nível acadêmico o debate sobre a qualidade de serviços, as ações de saúde e a inovação, estimulando a compreensão das relações entre eles. Em nível organizacional, a contribuição apresenta um diagnóstico local da inovação no contexto das ações de atenção à saúde, demonstrado pela relação das variáveis a serem gerenciadas, para o desenvolvimento adequado dos recursos sanitários locais e a promoção da qualidade de vida para a população adscrita.
\end{abstract}

Palavras-chave: inovação; PCATool; atenção primária.

Relación entre la innovación y la orientación hacia la calidad del servicio de salud de atención primaria

La innovación juega un diferencial en respuesta a las necesidades globales de la salud, ya que representa el cambio que impulsa el desarrollo de las organizaciones y conduce a una mayor eficiencia de sus procesos a través de la asignación adecuada de los recursos. El artículo es una contribución, resalte el debate académico a nivel de la calidad de los servicios y acciones de salud y estimular la innovación entender las relaciones entre ellos. A nivel organizativo la contribución presenta un locus diagnóstico de la innovación en el contexto de las acciones de atención de la salud, demostrado por la relación de las variables que deben gestionarse para el desarrollo adecuado de los recursos de salud, y la promoción de la calidad de vida de la población inscrita.

Palabras clave: innovación; PCATool; atención primaria.

Artigo recebido em 23 nov. 2011 e aceito em 16 maio 2013.

Rev. Adm. Pública - Rio de Janeiro 47(5):1243-264, set./out. 2013 


\begin{abstract}
Relationship between innovation and quality orientation of the health service for primary care Innovation plays a differential in response to global health needs, as it represents the change that encourages the development of organizations and leads to greater efficiency of its processes through the appropriate allocation of resources. The article is a contribution; highlight academic-level debate on the quality of services, health actions and innovation stimulating understanding the relationships between them. At the organizational level the contribution presents a diagnosis locus of innovation in the context of the actions of health care, demonstrated by the relationship of the variables to be managed for the proper development of health resources, and the promotion of quality of life for the enrolled population.
\end{abstract}

KEYwords: innovation; PCATool; primary care.

\title{
1. Introdução
}

A Constituição Federal brasileira de 1988 preconiza a amplitude do direito à saúde com o objetivo de atingir o bem-estar e o bem social, por meio da garantia de acesso universal às ações e aos serviços de saúde, contextualizados na municipalização. Assim, de acordo com Pereira e colaboradores (2003), desde a Primeira Conferência Nacional de Ciência e Tecnologia em Saúde, em 1994, a estrutura administrativa do governo e das políticas públicas tem direcionado esforços para o desenvolvimento da inovação, com o objetivo de assegurar as premissas legais.

Inseridas na municipalização, encontram-se as ações, propriamente ditas, organizadas sob a forma de redes integradas de saúde, apresentando como núcleo gerenciador a atenção primária, que, de acordo com Starfield (2002), representa uma forma de organização dos serviços de saúde que responde a uma estrutura assistencial com valores, princípios e elementos próprios. Da mesma forma, age como um regulador ao consumo indiscriminado e mercadológico dos serviços de saúde, se caracterizando como a porta de entrada ao sistema de saúde para as ações de saúde.

A inovação é descrita por Tidd e colaboradores (2005) como o processo-chave associado à renovação dentro da organização ao reanimar o que ela oferece e como cria e distribui esta oferta. Assim, as organizações são obrigadas a inovar para adquirirem um diferencial, o que sugere uma dinâmica organizacional em uma complexa rede de relações, de produção de conhecimento, como fatores essenciais para o desenvolvimento econômico e social de uma determinada região (Tigre, 2006). As organizações inovadoras são as que oferecem novos produtos e adotam ou põem em marcha novos processos ou serviços como meio de agregar valor e reduzir custos.

De acordo com o Manual de Oslo (2005), a inovação pode ocorrer em qualquer setor da economia, incluindo serviços governamentais, como saúde e educação, e representa um sinônimo de mudança. Dessa forma, este artigo explora a possibilidade de integração entre as dimensões da inovação e o estado da arte da atenção primária no município de Caxias do Sul 
(RS). Para tanto, foram destacadas algumas variáveis de análise da estrutura organizacional do serviço da atenção primária, sob a ótica dos temas de inovação.

Este artigo foi dividido em quatro seções, além da seção introdutória. A seção dois apresenta o referencial teórico de aproximação entre as variáveis de pesquisa sobre atenção primária e inovação. A seção três aponta os principais direcionadores metodológicos da pesquisa. A seção quatro apresenta a análise e a discussão dos resultados do estudo de caso, sob um enfoque qualitativo e quantitativo. E, por fim, a seção cinco apresenta as considerações finais da pesquisa a partir da análise da relação da inovação e da qualidade da orientação do serviço de saúde para atenção primária de Caxias do Sul.

\section{Referencial teórico}

\section{Inovação}

Desde o lançamento da obra Teoria do desenvolvimento econômico, de Joseph Schumpeter, em 1912, o tema inovação vem sendo exaustivamente analisado, debatido e teorizado. Segundo Schumpeter (1934), nas economias capitalistas, o desenvolvimento econômico é dirigido pelo impacto das inovações tecnológicas, que ocorrem por meio de um processo dinâmico, denominado "destruição criadora", no qual as novas tecnologias substituem as antigas, contrapondose, assim, à ideia do equilíbrio geral da economia, tal como descrito na teoria neoclássica.

Drucker (1981) e Toivonen e Tuominen (2009) afirmam que grande parte dos estudos sobre inovação enfoca o aspecto tecnológico e evidenciam que a inovação não é, apenas, um termo técnico, referindo-se, também, às dimensões econômicas e sociais. Ven (1999) complementa que a inovação é um processo de desenvolvimento e implantação de uma novidade, incluindo novos processos ou o desenvolvimento de novas ideias, como uma nova tecnologia, produto, processo ou novos arranjos.

De acordo com Dosi (1982), a inovação diz respeito à busca, à descoberta, à experimentação e à adoção de novos produtos, processos e novas formas organizacionais. Assim, a inovação constitui-se em um processo de busca e aprendizado, é socialmente determinada, e fortemente influenciada por formatos institucionais e organizacionais específicos, tais como a diversidade regional, especificidades locais etc. Corroborando, Miedzinski (2006) ressalta que os processos de inovação são chaves para o desenvolvimento, avanço econômico e social da região, através de relações de diversos setores e atores, agindo de forma complementar no incentivo ao desempenho da economia e ao bem-estar social.

Consoante a ampla bibliografia sobre estudos da inovação e seus distintos enfoques, Potts e Kastelle (2010) definem a inovação como o conjunto de atividades inscritas em um determinado período de tempo e lugar, que conduzem à introdução com êxito no mercado, e, pela primeira vez, de uma ideia, o que pressupõe a adoção de novos ou melhores produtos, processos, serviços ou técnicas de gestão e organização. Corroborando, para Hesselbein (2002), a inovação é identificada como uma mudança, que cria uma nova dimensão do de- 
sempenho. Essa mudança pode originar-se de uma nova ideia, que pode ser resultante de uma recombinação de velhas ideias, num esquema que desafia a ordem presente, ou seja, uma fórmula ou abordagem que é percebida como nova pelos indivíduos envolvidos.

Assim, considerando a trajetória bibliográfica, pode-se afirmar que a inovação é vista como um processo de mudança. Tidd e colaboradores (2005) e Bessant e Tidd (2007) classificam a inovação em quatro diferentes dimensões, corroboradas, também, pelo Fórum de Inovação da Escola de Administração de Empresas de São Paulo, da Fundação Getulio Vargas (Leite, 2005): a) inovação de produtos e/ou serviços relacionada a mudanças de um produto ou serviço oferecido pela organização; b) inovação de processos relacionada a mudanças no modo através do qual os produtos ou serviços são criados e distribuídos; c) inovação de gestão (organizacional — processo mental) relacionada a mudanças nos modelos mentais subjacentes que moldam o que a organização faz; e d) inovação de marketing (posição competitiva) relacionada a mudanças no contexto em que os produtos ou serviços são introduzidos no mercado. Estas categorias, também, estão descritas no Manual de Oslo (2005), que consiste em um instrumento que tem por objetivo fixar diretrizes para coleta e interpretação de dados sobre inovação, fazendo parte de uma série de publicações da Organização para Cooperação e Desenvolvimento Econômico (OCDE), apresentando, desta forma, uma maior compreensão das classificações de inovação.

Independentemente da análise sob os aspectos da inovação e sua ênfase, originalmente, tecnológica, Tidd e colaboradores (2005) esclarecem que a inovação por si e os processos de inovação não se caracterizam como algo simples e pontual, como uma sequência de atividades, mas sim como um processo complexo, recursivo, que ocorre em ciclos de variação, seleção e retenção determinando uma natureza constantemente dinâmica.

Assim, recorremos nessa pesquisa a uma definição seminal dos conceitos da inovação, como já exposto, procurando focar nossa análise nos serviços de saúde. Assim, qualquer mudança no comportamento pretendido, em determinado nível institucional, sendo uma disposição nova ou melhorada, um procedimento tecnológico, ou ferramenta administrativa que propiciem uma variação da prestação dos serviços de saúde, ou, ainda, instrumentos necessários à sua prestação, serão estudados sob a ótica da tipologia proposta pelo Manual de Oslo (2005).

\section{Atenção primária}

A atenção primária é uma concepção de sistema de saúde, de acordo com Starfield (2002). No Brasil, pode-se dizer que o sistema de saúde está perfeitamente afinado com a atenção primária, uma vez que são identificados valores e princípios essenciais no estabelecimento das prioridades nacionais em saúde, conforme a Constituição Federal do Brasil de 1988. Em outras palavras, trata-se de um Sistema Único de Saúde (SUS), voltado a enfatizar a equidade social, a universalidade e a integralidade à saúde (Mendes, 2007). 
A Organização Mundial da Saúde (OMS) está empenhada em espalhar os elementos de consolidação da atenção primária até os dias atuais, ou seja, a integração dos serviços locais, a participação social, a necessidade de ações intersetoriais e a relação da saúde com o desenvolvimento econômico e social. Corroborando a importância da melhoria de ações e serviços de saúde condizentes à população, o Relatório do Banco Mundial (World Bank, 1993) sistematizou alguns pontos, nos quais as melhorias em saúde influenciam o crescimento econômico. Destacaram-se os seguintes: a) ganhos na produtividade do trabalho; b) melhor utilização de recursos naturais; c) benefícios oriundos da educação para as próximas gerações; d) redução nos custos da assistência médica; e) influência dos investimentos em saúde sobre a redução da pobreza.

Ademais, a atenção primária é representada como uma associação de diversos atores e setores na produção de serviços públicos de saúde (Gauld et al., 2012), em conformidade com uma rede de interações. Albuquerque e Cassiolato (2000) destacam que a saúde pública entra com um papel importante nesse complexo setor, tendo interações diretas com as universidades, instituições de pesquisa, além de conceber as inovações de complexo de relações intersetoriais. Existe um ambiente propício para a inserção das inovações, considerando que a efetividade das inovações, implementadas pela saúde pública, repercute diretamente sobre o bem-estar da população.

Assim, as redes podem formar uma resposta para reduzir a incerteza e o grau de irreversibilidade do processo de inovação, racionalizando o custo e os riscos do desenvolvimento de um novo campo de conhecimento, aumentando, assim, a flexibilidade e a reversibilidade dos comprometimentos. Isto é reforçado por autores que analisam o paradigma da organização em rede para a inovação nas aglomerações regionais (Cooke e Morgan, 1998; Rothwell, 1996). Segundo esses autores, a rede seria uma forma mais eficiente de dar conta da complexidade e da incerteza presente no processo de inovação.

No contexto local, a atenção primária deve cumprir, nas redes de atenção à saúde, três funções: a função de resolução, a função de coordenação e a função de responsabilização, para que todo o sistema funcione de forma eficiente (Conass, 2007). A atenção primária influencia na melhoria dos indicadores de saúde e age como grande potencial regulador da utilização dos recursos de alta densidade tecnológica, garantindo o acesso universal aos serviços que tragam reais benefícios à saúde da população (Bodenheimer, 2006). Dessa forma, ressalta-se o aspecto fundamental da otimização dos serviços de saúde sob a estrutura da atenção primária como forma de garantia de prestação de serviço consoante as tendências identificadas no cenário de evolução temporal da atenção à saúde de diversos países, como o crescimento populacional, a urbanização, o envelhecimento demográfico e os aumentos de gastos com saúde.

Nessa perspectiva, Gadelha e colaboradores (2007) assinalam a necessidade de "tratamento das inovações em saúde em articulação e equilíbrio com as dinâmicas econômicas, das instituições e da política pública". O autor justifica essa necessidade porque a área da saúde constitui um dos espaços econômicos mais dinâmicos de acumulação de capital e de 
inovação, cuja compreensão se mostra essencial para pensar políticas de promoção e de desenvolvimento.

Decorrente das evidências crescentes de que a atenção primária contribui de forma decisiva para melhorar os resultados de saúde da população, Starfield e colaboradores (2001) desenvolveram e validaram um instrumento denominado Primary Care Assessment Tool (PCATool) ${ }^{1}$ como forma de avaliação de estrutura e processos prestados dos serviços na área da saúde. Estudos, no Brasil, desenvolvidos por Harzheim e colaboradores (2006), validaram o instrumento, versão infantil e adulto, em um processo envolvendo a tradução para o português, tradução reversa, do inglês para o português, debriefing (aplicação prévia do questionário para indivíduos semelhantes aos da população em estudo para avaliar seu grau de compreensão), validação do conteúdo e constructo, e avaliação da consistência interna e confiabilidade (ressaltando a precisão e a estabilidade ao longo do tempo) por análises estatísticas.

Baseado nesse referencial, em 2010, o Ministério da Saúde, publicou, oficialmente, o Manual do Instrumento de Avaliação da Atenção Primária à Saúde (PCATool — Brasil), evidenciando a sua utilidade, principalmente, para a realização de investigações científicas pertencentes ao contexto acadêmico de construção de conhecimento, como também no contexto cotidiano da prática e gestão da atenção primária (Brasil, 2010).

Esse questionário avalia o quanto os serviços de saúde estão orientados para os atributos definidores da atenção primária, em quatro atributos essenciais e três atributos derivados, respectivamente: a) acesso de primeiro contato, que envolve o acesso aos serviços pela população para cada nova necessidade, observando quantidade de pessoas da qual a unidade é responsável e período em que a unidade está acessível aos usuários; b) longitudinalidade ou atendimento continuado, que envolve a atenção orientada para a pessoa, fornecida ao longo do tempo (mínimo de dois anos), e vínculo com a unidade prestadora do serviço; c) integralidade, que envolve a especificação da variedade de serviços disponíveis e serviços prestados para as necessidades da população, exceto as incomuns, que são de responsabilidade do atributo derivativo da competência cultural; d) coordenação, que envolve a capacidade de integração de pessoas e sistema de informação para os serviços oferecidos onde quer que tenham sido recebidos; e) orientação familiar ou enfoque na família, que envolve o conhecimento e o acompanhamento familiar dos problemas de saúde; f) orientação comunitária, que envolve o conhecimento da comunidade atendida, a participação em atividades comunitárias e o envolvimento da comunidade na unidade prestadora; e g) competência cultural, que envolve a capacitação gerencial à frente do serviço de saúde e também a prestação de serviços especiais à população.

Assim, a partir do referencial teórico, foi possível destacar, no quadro 1, a aproximação da dimensão das variáveis dos estudos sobre a inovação e o escore do PCATool, que passam a ser o objetivo desta pesquisa.

1 (C)1998 Primary Care Policy Center for Underserved Populations Johns Hopkins University, 1998. 
Quadro 1

\section{Variáveis da pesquisa}

\begin{tabular}{|c|c|c|c|}
\hline \multirow{8}{*}{ 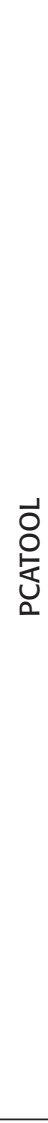 } & Variáveis de Pesquisa & Conceitos Evidenciados & Fonte Consultada \\
\hline & Acesso & $\begin{array}{l}\text { Acessibilidade da unidade } \\
\text { Acesso à atenção } \\
\text { Uso da unidade como local de primeiro contato }\end{array}$ & \multirow{7}{*}{$\begin{array}{l}\text { Starfield (1994), } \\
\text { Starfield, Shi e Jiahong } \\
\text { (2001), } \\
\text { Vuori (1992), } \\
\text { World Health Organi- } \\
\text { zation (2005), } \\
\text { Mendes (2007), } \\
\text { Macinko e Almeida } \\
\text { (2004), } \\
\text { Gofin, Gofin e Cueto } \\
\text { (2005), } \\
\text { Health Evidence } \\
\text { Network (2004), } \\
\text { Den Exter e colabora- } \\
\text { dores (2004), } \\
\text { Brasil (2010) }\end{array}$} \\
\hline & Longitudinalidade & $\begin{array}{l}\text { Definição da população eletiva } \\
\text { Conhecimento do paciente e do seu meio social } \\
\text { Extensão e força da relação com os pacientes }\end{array}$ & \\
\hline & Coordenação & $\begin{array}{l}\text { Mecanismos para a continuidade } \\
\text { Reconhecimento de informações e consultas prévias e } \\
\text { de encaminhamento }\end{array}$ & \\
\hline & Integralidade & $\begin{array}{l}\text { Espectro de serviços disponíveis } \\
\text { Atividades preventivas primárias e secundárias } \\
\text { Reconhecimento e manejo dos problemas de saúde }\end{array}$ & \\
\hline & Orientação Familiar & $\begin{array}{l}\text { Conhecimento dos membros da família e dos proble- } \\
\text { mas de saúde da família }\end{array}$ & \\
\hline & $\begin{array}{l}\text { Orientação Comu- } \\
\text { nitária }\end{array}$ & $\begin{array}{l}\text { Conhecimento das necessidades de saúde da comu- } \\
\text { nidade } \\
\text { Participação nas atividades comunitárias }\end{array}$ & \\
\hline & Competência Cultural & $\begin{array}{l}\text { Atendimento às necessidades especiais associado às } \\
\text { características culturais } \\
\text { Prestação de serviços especiais para atender as neces- } \\
\text { sidades culturais }\end{array}$ & \\
\hline \multirow{4}{*}{ 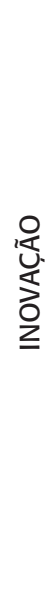 } & $\begin{array}{l}\text { Produto (Bem ou } \\
\text { Serviço) }\end{array}$ & $\begin{array}{l}\text { Introdução de um bem ou serviço novo ou significati- } \\
\text { vamente melhorado no que concerne a suas característi- } \\
\text { cas ou usos previstos. Incluem-se melhoramentos significa- } \\
\text { tivos em especificações técnicas, componentes e materiais, } \\
\text { facilidade de uso ou outras características funcionais. }\end{array}$ & \multirow{4}{*}{$\begin{array}{l}\text { Manual de Oslo } \\
\text { (2005), } \\
\text { Ahmed (2001), } \\
\text { Damanpour, Szabat e } \\
\text { Evan (1989), } \\
\text { Hesselbein (2002), } \\
\text { Higgins (1995), } \\
\text { Malerba (1999), } \\
\text { Tidd, Bessant e Pavitt } \\
\text { (2005) }\end{array}$} \\
\hline & Processo & $\begin{array}{l}\text { Implantação de um método de produção ou distri- } \\
\text { buição novo ou significativamente melhorado. Incluem-se } \\
\text { mudanças significativas em técnicas e equipamentos. }\end{array}$ & \\
\hline & Marketing & $\begin{array}{l}\text { Implantação de um método de marketing com mu- } \\
\text { danças significativas na concepção do produto ou em sua } \\
\text { embalagem, no posicionamento do produto, em sua pro- } \\
\text { moção ou na fixação de preços. }\end{array}$ & \\
\hline & Organizacional & $\begin{array}{l}\text { Implantação de um método organizacional nas práti- } \\
\text { cas de negócio da instituição, na organização de seu local } \\
\text { de trabalho ou em suas relações externas. }\end{array}$ & \\
\hline
\end{tabular}

Fonte: Elaboração própria. 
Assim, evidencia-se que a atenção primária deve apresentar uma variedade de serviços, garantindo o acolhimento da população adscrita e, consequentemente, prestando um atendimento continuado, coordenando fluxos de referência e contrarreferência para outros níveis do sistema de saúde.

Mendes (2007) estabelece que as Unidades Básicas de Saúde (UBS) representam o local de consolidação da comunicação da atenção primária, pois se entende que corresponde à primeira prestação do serviço de saúde e, consequentemente, do primeiro contato com os problemas de saúde da população. No Brasil, a organização da atenção primária está delegada à gestão municipal e os repasses de recursos federais são automáticos. Atualmente, a principal estratégia de revitalização da atenção primária, no Brasil, é a Estratégia da Saúde da Família (ESF), implantado em 1994, que tem recebido importantes incentivos financeiros visando à ampliação da cobertura populacional e à reorganização da atenção.

Portanto, evidencia-se que o setor da saúde é visto como um complexo de relações intersetoriais que propiciam inovações que estão em articulação com as dinâmicas econômicas, das instituições e das políticas públicas, representando um espaço de grande acumulação de conhecimento para promoção de políticas e de desenvolvimento local (Gadelha et al., 2007). Assim, constata-se que o desenvolvimento dessa estrutura do sistema de saúde pode auxiliar no aperfeiçoamento das ações e prestação de serviços à população e, consequentemente, um auxílio no desenvolvimento econômico e social da região.

\section{Metodologia}

A construção da base conceitual serviu para a consolidação do referencial teórico utilizado a partir da revisão bibliográfica de pesquisas e estudos já realizados na área em questão. Especificamente, este artigo apresenta uma análise a partir de dados provenientes de uma pesquisa prévia sobre a relação de variáveis que definem a qualidade de um sistema de saúde orientado para atenção primária e a tipologia das inovações, no município de Caxias do Sul (RS).

Os elementos utilizados para análise foram construídos a partir de um estudo de caso em duas fases, qualitativo e quantitativo. A fase qualitativa teve por objetivo explorar e comparar a teoria com a prática, proporcionando uma maior familiaridade com o problema. De acordo com Yin (2005), para o levantamento de dados qualitativos utilizaram-se os seguintes instrumentos de coleta: a) pesquisa bibliográfica; b) registro em arquivos da Secretaria Municipal de Saúde de Caxias do Sul; c) entrevistas semiestruturadas aos gestores das UBS; e e) observação e análise de outros documentos, como atas de reuniões e pareceres. Assim foi possível identificar a quantidade de inovações de acordo com a classificação do Manual de Oslo (2005). Esses dados foram tratados mediante a análise de conteúdo, que, conforme Bardin (2006), se define como um conjunto de técnicas de análise das comunicações visando obter, por procedimentos sistemáticos e objetivos de descrição do conteúdo das mensagens, 
indicadores (quantitativos ou não) que permitam a inferência de conhecimentos relativos às condições de produção (variáveis inferidas) destas mensagens.

$\mathrm{Na}$ fase quantitativa, foi utilizado o questionário com perguntas fechadas, que possibilitaram a garantia da análise do grau com que as medições estão isentas de erros aleatórios, coletadas a partir dos indivíduos. Yin (2005) aponta que a pesquisa quantitativa é um método de pesquisa social que utiliza técnicas estatísticas que, normalmente, implicam a construção de inquéritos por questionário. O questionário denominado PCATool contém 77 questões que possibilitaram a identificação e a quantificação dos atributos essenciais e derivados que orientam a qualificação da atenção primária. Os escores são construídos para cada atributo a partir da média aritmética das respostas e então padronizados numa escala de zero a 10. A média dos escores por atributo fornece um escore geral da orientação do serviço para atenção primária. Este escore foi classificado com o limiar de 6,6. Se fosse superior a esse valor, representaria um alto escore; se inferior a esse valor, seria classificado como baixo escore, com base no referencial teórico.

Ressalta-se que o instrumento de coleta de dados, composto pela entrevista semiestruturada e o questionário, foi aplicado sob forma de um pré-teste a especialistas e, posteriormente, validado junto à Secretaria Municipal da Saúde do município, a fim de se verificarem a clareza de informações descritas, o entendimento e a coerência das respostas obtidas. Além disso, os resultados referentes à classificação das inovações também obtiveram validação com especialistas da Secretaria Municipal de Saúde (SMS) de Caxias do Sul (Nodari et al., 2012).

Assim, da população de 41 UBS que compõem o Sistema de Saúde de Caxias do Sul selecionou-se uma amostra de 15 UBS, todas possuidoras de ESF. As características gerais das UBS selecionadas encontram-se resumidas na tabela 1.

A metodologia empregada neste artigo foi de um estudo exploratório e descritivo. Para a análise dos dados propôs-se uma abordagem qualitativa e quantitativa. Por meio das técnicas qualitativas torna-se possível interpretar os resultados, comprovando-os através das técnicas quantitativas (Alves-Mazzotti e Gewandsznajder, 1999) que, corroborando Flick (2004), não são opostos incompatíveis que não podem ser combinados.

$\mathrm{Na}$ fase quantitativa da pesquisa, foram realizadas análises estatísticas do cálculo do coeficiente de correlação linear de Pearson (r), coeficiente de determinação $\left(r^{2}\right)$ e de regressão linear, a fim de se identificar a força da relação entre as variáveis selecionadas para este estudo e a influência de uma variável sobre a outra (Hair Jr. et al., 2006). Consideraram-se como variáveis dependentes a inovação de produto, processo, marketing e organizacional. As variáveis independentes foram acesso, longitudinalidade, coordenação, integralidade, orientação familiar, orientação comunitária e a competência cultural.

A tabulação e o processamento dos dados de estatística inferencial foram realizados a partir do suporte informático do pacote estatístico SPSS Versão 19. S. 
Tabela 1

Características gerais das UBS pesquisadas

\begin{tabular}{|cccccccc|}
\hline UNIDADE & $\begin{array}{c}\text { Número da } \\
\text { população } \\
\text { cadastrada } \\
\text { (Cartão SUS) }\end{array}$ & $\begin{array}{c}\text { Total de } \\
\text { atendimentos } \\
\text { das UBS }\end{array}$ & $\begin{array}{c}\text { Quantidade } \\
\text { de equipes de } \\
\text { ESF }\end{array}$ & $\begin{array}{c}\text { Número de } \\
\text { atendimento } \\
\text { ESF }\end{array}$ & $\begin{array}{c}\text { Total de } \\
\text { funcionários** }\end{array}$ & $\begin{array}{c}\text { Total de despesas } \\
\text { por UBS (R\$)** }\end{array}$ \\
\hline UBS1 & 1.090 & 8.074 & 1 & 2.130 & 6 & 270.698 \\
UBS2 & 745 & 7.123 & 1 & 2.439 & 5 & 166.662 \\
UBS3 & 3.976 & 12.376 & 1 & 2.546 & 7 & 137.974 \\
UBS4 & 3.653 & 10.345 & 1 & 4.862 & 8 & 272.089 \\
UBS5 & 14.718 & 89.101 & 3 & 41.877 & 32 & 2.716 .994 \\
UBS6 & 3.586 & 22.118 & 2 & 10.395 & 11 & 669.459 \\
UBS7 & 11.169 & 51.116 & 3 & 24.025 & 21 & 1.438 .619 \\
UBS8 & 7.678 & 34.453 & 2 & 16.193 & 19 & 864.729 \\
UBS9 & 11.411 & 44.288 & 3 & 20.815 & 24 & 1.617 .125 \\
UBS10 & 4.152 & 9.092 & 1 & 4.273 & 7 & 340.467 \\
UBS11 & 6.637 & 24.361 & 2 & 11.450 & 15 & 922.073 \\
UBS12 & 7.220 & 27.278 & 2 & 12.821 & 15 & 988.758 \\
UBS13 & 1.800 & 23.460 & 2 & 11.026 & 8 & 724.273 \\
UBS14 & 5.667 & 30.458 & 2 & 14.315 & 13 & 921.523 \\
UBS15 & 1.847 & 6.776 & 1 & 3.185 & 5 & 299.419 \\
\hline
\end{tabular}

Fonte: Nodari e colaboradores (2012).

* Estatutários e celetistas.

** Expresso em milhares de reais $(\mathrm{R} \$)$.

\section{Análise e discussão dos resultados}

\section{Características da atenção primária de Caxias do Sul}

Segundo o IBGE (2013), Caxias do Sul possui um Produto Interno Bruto (PIB) em serviços de $\mathrm{R} \$ 7$ milhões, sendo o setor industrial responsável por $\mathrm{R} \$ 6$ milhões, ficando em segundo lugar no PIB estadual. Possui uma expectativa de vida ao nascer de 74,11 anos e um PIB per capita (2010) de $\mathrm{R} \$ 36$ mil. A relevância da escolha do objeto de estudo, do município de Caxias do Sul (RS), se justifica pelo investimento crescente no decorrer da última década na saúde pública, com incrementos de aproximadamente $16 \%$, a cada ano, nas despesas totais do município (Brasil, 2011). Ademais, se justifica pela precoce introdução de melhores práticas 
de organização da APS nas características de serviços, constituindo um polo de referência no setor saúde da região nordeste do estado do Rio Grande do Sul (Claus e Capra, 2002).

Historicamente, o município de Caxias do Sul se deparava com a insuficiência de ofertas de ações e serviços públicos de saúde, com a baixa inserção da saúde pública em relação ao setor privado e com a fragilidade das estruturas de gestão, sendo, gradativamente, substituído pela decisão de investimentos significativos e a definição de uma proposta político-assistencial, para o SUS municipal, a partir de 1997. Os principais problemas levantados quanto à saúde pública, identificados nesse período, no município, de acordo com Claus (2007), eram: a) atenção assistencial centrada nas tarefas e nos procedimentos profissionais, e não nas necessidades dos usuários; b) baixa efetividade e eficiência das ações em saúde; c) planejamento de ações e recursos centralizados, com inexistência de planos locais de saúde; d) dificuldades de vinculação de usuários aos serviços básicos de referência; e) dificuldade de acesso dos usuários dos serviços; f) pouca responsabilização das equipes de saúde em relação à saúde da população de sua área de abrangência; e g) concepções difusas sobre a missão da UBS e dos demais serviços de apoio.

A definição de uma nova proposta político-assistencial, no município de Caxias do Sul, representou uma concepção de organização do sistema de serviços de saúde, denominada pelo município "Em Defesa da Vida", que resultava na operação em rede com características horizontais e não mais uma estrutura piramidal, como tradicionalmente vinha sendo concebida a hierarquização de serviços. Dessa forma, a logística do SUS e a lógica da atenção primária, denominada atenção à saúde, fundamentaram-se a partir de sua célula principal — a UBS - , unidade mediadora de todo o sistema de saúde, e os demais serviços ambulatoriais e hospitalares, de média e alta complexidade, cumprindo o papel de apoio e retaguarda para que a rede básica concretize a sua missão (Caxias do Sul, 2004).

O documento denominado "Protocolo de Ações Básicas de Saúde - Uma proposta em Defesa da Vida" desenvolveu as definições de uma proposta assistencial compreendendo um conjunto de concepções e ações necessárias ao monitoramento das condições de saúde e intervenção nas intercorrências da população do município. Ressalta-se que esse documento, publicado em 2000, previamente à deliberação de estratégias gerais da esfera federal de controle da atenção primária no Brasil, foi premiado como trabalho inédito em Experiências Inovadoras do Ministério da Saúde (Claus, 2007).

A partir das 41 UBS distribuídas pelo município, a rede atual permite o acesso a serviços de média e alta complexidade, que antes não eram acessíveis aos usuários do SUS, e realiza uma média de 15 mil consultas/mês. A população demandante de serviços de saúde é cadastrada de acordo com as diretrizes do SUS através do Cartão SUS e deve receber atendimento na UBS mais próxima e pela ESF de acordo com a localização de sua residência. Em outubro de 2009, o banco de dados do Cartão SUS do município de Caxias do Sul contava com 340 mil usuários cadastrados.

Em 2004 foi implantada no município a Estratégia da Saúde da Família (ESF), que tem sido aplicada, no Brasil, como um meio de auxílio na regulamentação da política pública de atenção primária. O município de Caxias do Sul apresentava, em 2009, 27 ESF distribuídas em 15 UBS. 
De acordo com a Secretaria de Desenvolvimento Econômico (2008), a saúde pública constitui o primeiro orçamento do município. Atualmente, a rede de UBS local possibilita à população residente no município ter o acesso a consultas nas áreas de clínica, ginecologia, obstetrícia, pediatria e enfermagem, além de medicamentos, os quais constituem uma lista de aproximadamente 118 itens selecionados segundo critérios estabelecidos pelo Ministério da Saúde, a partir do perfil epidemiológico da população.

De acordo com Claus (2007), o município de Caxias do Sul criou em 1998 a função do gerente de UBS. Campos (1992) afirma que o trabalho de gerência desenvolvido em uma UBS pode ser concebido como um meio para se consolidar um determinado processo de produção de ações em saúde ou para transformá-los.

Para as UBS, foi definida a missão de ser o centro do sistema de saúde, realizando a assistência qualificada com vigilância à saúde da população. Além disso, é através da UBS que acontecem os fluxos de encaminhamento de referência e contrarreferência para os outros níveis da rede de atenção à saúde.

Assim, o sistema de saúde de Caxias do Sul, com o centro de comunicação na atenção primária, operacionalizada através da UBS, corresponde a uma organização horizontal com fluxos de referência e contrarreferência regulada pela demanda da população, que conta com centros denominados: a) sistemas de apoio, compostos por serviços de farmácia, internação hospitalar, hemodiálise, consultas especializadas; b) sistemas logísticos, compostos por sistema de informações de marcação de consultas, ambulâncias; e c) sistemas de gestão, composto pela regulação assistencial da Secretaria Municipal de Saúde (Nodari et al., 2012).

\section{Análise das Unidades Básicas de Saúde (UBS)}

As 15 UBS possuem uma estrutura física que compreende de $350 \mathrm{~m}^{2}$ a $500 \mathrm{~m}^{2}$ e horário de atendimento de $12 \mathrm{~h}$ por dia. Das UBS integrantes desse estudo, cinco estão localizadas na zona rural e 10 unidades estão localizadas na zona urbana do município de Caxias do Sul. Todas as UBS selecionadas possuem equipes de ESF. Ao gestor da UBS cabe a missão de conduzir técnica, política e administrativamente os processos de trabalho de forma a garantir as mudanças estratégicas elencadas para as necessidades locais do seu território de abrangência, ou seja, da UBS, e organizar as equipes da ESF.

As inovações introduzidas pelas 15 UBS, no período correspondente a janeiro de 2007 a outubro de 2009, foram 52 inovações no total: 24 foram inovações de produto (bem ou serviço,) representando 46\% do total de inovações, 15 inovações de processo, representando $29 \%$ do total de inovações, cinco inovações de marketing e oito inovações organizacionais, representando, respectivamente, $10 \%$ e $15 \%$ do total de inovações.

Dentre as principais inovações de produto destacam-se a introdução de exames e serviços de outras áreas da saúde, como nutrição e fisioterapia, além de programas visando à ampliação da oferta de atendimento para a população, com incentivo em associação com as esferas estadual, federal e demais secretarias municipais. 
Nas inovações de processo, identificaram-se mudanças significativas em técnicas, como a coleta de exames instituída no local da UBS. Outra inovação foi acerca do acolhimento de todos os usuários, visando o atendimento, garantindo os princípios de equidade e integralidade das ações em saúde. Essas mudanças proporcionaram o desenvolvimento de novos meios de processos, que permitiram maior presteza nos procedimentos de atendimento ao usuário e de entrega do serviço, como na coleta de exames.

As inovações de marketing foram as de menor quantidade e estão associadas a programas com comunidades ou de orientação da gestão da SMS, como o Vigimama, relacionado à saúde da mulher, com a identificação de nichos de atuação em um mesmo mercado. Ainda, essas inovações estão relacionadas com a mudança de comportamento da organização no mercado em que ela está inserida, como no caso das ouvidorias, disponíveis para a população das UBS visando à conscientização da população quanto à prevenção e ao tratamento das enfermidades, ao mesmo tempo que apreende as necessidades da população.

E, por fim, as inovações organizacionais que corresponderam à implantação de métodos organizacionais visando a melhoria das condições do local de trabalho, como o acolhimento específico para pediatria e mudanças de layout promovendo a absorção da população e, consequentemente, a satisfação no atendimento de seus problemas de saúde. Ademais, observou-se, ainda, a introdução de novos gerenciamentos de processos, como podemos observar com a informatização do Sistema de Informação - Saúde da Atenção Primária (SIS.SAP), movimento iniciado em 2009 e que, de acordo com a SMS, deverá ser implantado em todas as UBS em 2010, facilitando e permitindo a agilidade e a troca de informações no Sistema de Atenção à Saúde.

Na análise do PCATool avaliou-se a qualidade de orientação para atenção primária de Caxias do Sul. Identificou-se que a média do escore para as 15 UBS, integrantes do objeto de estudo, apresentou-se em 6,81 , o que representa um alto escore de atenção primária com um Coeficiente de Variação (CV) de 14,68\%. Constatou-se que cinco UBS não atingiram o valor central de 6,6, que representava o limite de escore de atenção primária proposto pela orientação dos estudos de Starfield e colaboradores (2001), sendo quatro UBS da zona rural e uma UBS da zona urbana. A tabela 2 apresenta as informações resumidas, referentes aos escores parciais do PCATool e ao número de inovações para cada UBS, que serviram de base para a análise estatística.

\section{Análise estatística dos dados coletados}

Partindo do conjunto de dados para as variáveis contínuas, realizaram-se medidas descritivas de dispersão e centralização, para determinar a média das características de cada uma das variáveis e sua linearidade, considerando a análise conjunta das 15 UBS. Assim, verificaram-se a força da relação das variáveis e a influência das variáveis independentes sobre as dependentes. Ressalta-se que foi realizada a transformação de valores discretos do número de 
inovações para valores contínuos a fim de possibilitar a análise proposta nessa pesquisa (Hair Jr. et al., 2006).

O coeficiente de correlação de Pearson (r) é uma medida de correlação linear entre duas variáveis aleatórias que examina a força de associação. Considerando a análise da variável número de inovações, e a variável escore parcial do PCATool, para as 15 UBS, foi possível identificar que, a partir das considerações do nível de significância de $5 \%$ (Valor- $\rho<0,05$ ), observou-se que o número de inovações apresenta uma forte relação positiva com o escore do PCATool de $r=0,769$ com Valor- $\rho=0,0008$. O coeficiente de determinação, que mede o modo de associação entre as variáveis, apresentou-se em $r^{2}=0,5915$, ou seja, indicou que, para cada unidade de mudança das variáveis contidas no escore do PCATool, se produz uma mudança de 0,5915 (59,15\%) no número de inovações.

Diante dos resultados de associação e força entre as variáveis, procurou-se, então, avaliar a influência de uma variável sobre a outra, estabelecendo uma relação de causa e efeito, por meio da regressão linear simples, ou seja, o quanto as variáveis contidas no PCATool, denominadas independentes, influenciam as variáveis do número de inovações, denominadas dependentes. A regressão linear simples constitui uma tentativa de estabelecer uma equação matemática linear que descreva o relacionamento entre duas variáveis (Hair Jr. et al., 2006). O método utilizado de avaliação para a regressão linear simples foi o dos mínimos quadrados.

Tabela 2

Variáveis do estudo do PCATool e inovação

\begin{tabular}{|c|c|c|c|c|c|c|c|c|c|c|c|c|c|c|c|c|}
\hline & $\begin{array}{c}\text { Variáveis de } \\
\text { Análise }\end{array}$ & $\begin{array}{c}\text { UBS } \\
1\end{array}$ & $\begin{array}{c}\text { UBS } \\
2\end{array}$ & $\begin{array}{l}\text { UBS } \\
3\end{array}$ & $\begin{array}{c}\text { UBS } \\
4\end{array}$ & $\begin{array}{l}\text { UBS } \\
5\end{array}$ & $\begin{array}{l}\text { UBS } \\
6\end{array}$ & $\begin{array}{l}\text { UBS } \\
7\end{array}$ & $\begin{array}{l}\text { UBS } \\
8\end{array}$ & $\begin{array}{c}\text { UBS } \\
9\end{array}$ & $\begin{array}{c}\text { UBS } \\
10\end{array}$ & $\begin{array}{c}\text { UBS } \\
11\end{array}$ & $\begin{array}{c}\text { UBS } \\
12\end{array}$ & $\begin{array}{c}\text { UBS } \\
13\end{array}$ & $\begin{array}{c}\text { UBS } \\
14\end{array}$ & $\begin{array}{c}\text { UBS } \\
15\end{array}$ \\
\hline \multirow{7}{*}{ 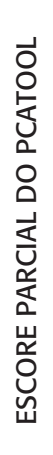 } & Acesso & 2,33 & 1,89 & 2,33 & 2,44 & 3,11 & 2,78 & 2,11 & 2,44 & 3,67 & 3,11 & 2,11 & 2,33 & 3,00 & 3,56 & 2,56 \\
\hline & Longitudinalidade & 2,38 & 2,62 & 2,38 & 2,62 & 3,00 & 2,85 & 2,46 & 3,38 & 3,38 & 3,23 & 2,46 & 3,00 & 3,23 & 4,00 & 2,54 \\
\hline & Coordenação & 2,86 & 2,62 & 2,54 & 2,93 & 3,54 & 2,93 & 2,79 & 3,35 & 3,10 & 2,62 & 2,60 & 3,03 & 3,22 & 3,15 & 2,94 \\
\hline & Integralidade & 3,32 & 2,71 & 3,18 & 3,32 & 3,63 & 3,31 & 2,98 & 3,70 & 3,33 & 2,71 & 3,16 & 3,53 & 3,25 & 3,19 & 3,25 \\
\hline & $\begin{array}{l}\text { Orientação } \\
\text { Familiar }\end{array}$ & 3,43 & 2,21 & 2,64 & 2,86 & 2,93 & 3,07 & 3,07 & 3,79 & 3,21 & 2,21 & 2,79 & 3,71 & 3,07 & 3,50 & 3,07 \\
\hline & $\begin{array}{l}\text { Orientação } \\
\text { Comunitária }\end{array}$ & 3,57 & 2,43 & 2,81 & 2,81 & 3,71 & 3,57 & 3,29 & 3,86 & 3,71 & 2,43 & 3,43 & 3,86 & 3,33 & 3,71 & 3,33 \\
\hline & $\begin{array}{l}\text { Competência } \\
\text { Cultural }\end{array}$ & 2,57 & 2,14 & 2,14 & 2,57 & 3,43 & 3,43 & 3,71 & 3,71 & 3,57 & 2,14 & 3,71 & 3,29 & 3,14 & 3,00 & 3,14 \\
\hline \multirow{4}{*}{ 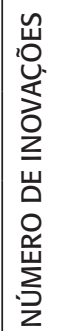 } & $\begin{array}{l}\text { Produto (Bem } \\
\text { ou Serviço) }\end{array}$ & 3 & 1 & 2 & 3 & 8 & 7 & 7 & 6 & 7 & 4 & 4 & 6 & 2 & 5 & 2 \\
\hline & Processo & 3 & 1 & 1 & 2 & 8 & 6 & 6 & 4 & 2 & 1 & 3 & 3 & 6 & 4 & 4 \\
\hline & Marketing & 1 & 1 & 0 & 1 & 2 & 3 & 2 & 2 & 2 & 1 & 1 & 2 & 2 & 2 & 3 \\
\hline & Organizacional & 1 & 1 & 2 & 1 & 3 & 2 & 3 & 3 & 2 & 1 & 2 & 1 & 3 & 3 & 1 \\
\hline
\end{tabular}

Fonte: Elaboração própria. 
A tabela 3 resume os valores do $r, r^{2}$ e $r^{2}$ ajustado do cruzamento das 11 variáveis. $\mathrm{O}$ coeficiente de correlação (r) é a medida da relação linear entre duas ou mais variáveis que indica a proximidade dos pontos à reta de regressão; quanto mais próximo estiver de um, mais próximos os pontos se encontram da reta de regressão; quanto mais próximo de zero, mais pobre é o ajustamento da reta de regressão de pontos (Hair Jr. et al., 2006). Assim, foi possível identificar na tabela 3 que as variáveis dependentes do número de inovações de produto, processo, marketing e organizacional possuem uma força de relação e associação com as variáveis independentes do escore do PCATool de coordenação, integralidade, orientação comunitária e orientação cultural.

O coeficiente de determinação $\left(\mathrm{r}^{2}\right)$ da regressão indica o quanto as variáveis independentes explicam a variação da variável dependente, ou seja, uma medida que procura refletir o quanto os valores dependentes estão relacionados com os independentes, podendo variar de zero a um, de modo que, quanto mais próximo de um, melhor o ajuste (Hair Jr. et al., 2006). Podemos observar na tabela 3 que, em relação à variável dependente inovação de produto, o maior coeficiente de determinação apresentou-se em $\mathrm{r}^{2}=0,466$ referente à variável independente competência cultural, ou seja, indica que, para cada unidade de mudança de competência cultural, se produziu uma unidade de mudança de 0,466 (46,60\%) na inovação de produto.

\section{Tabela 3}

Regressão linear simples para as variáveis da inovação e PCATool

\begin{tabular}{|c|c|c|c|c|c|c|c|c|c|c|c|c|}
\hline $\begin{array}{l}\text { Variável } \\
\text { Dependente }\end{array}$ & \multicolumn{3}{|c|}{ Produto } & \multicolumn{3}{|c|}{ Processo } & \multicolumn{3}{|c|}{ Marketing } & \multicolumn{3}{|c|}{ Organizacional } \\
\hline $\begin{array}{l}\text { Variável } \\
\text { Independente }\end{array}$ & $r$ & $r^{2}$ & $\begin{array}{c}\mathrm{r}^{2} \\
\text { Ajustado }\end{array}$ & $r$ & $r^{2}$ & $\begin{array}{c}r^{2} \\
\text { Ajustado }\end{array}$ & $\mathrm{r}$ & $r^{2}$ & $\begin{array}{c}\mathrm{r}^{2} \\
\text { Ajustado }\end{array}$ & $\mathrm{r}$ & $r^{2}$ & $\begin{array}{c}r^{2} \\
\text { Ajustado }\end{array}$ \\
\hline Acesso & 0,453 & 0,205 & 0,144 & 0,197 & 0,393 & $-0,35$ & 0,390 & 0,152 & 0,082 & 0,335 & 0,112 & 0,045 \\
\hline Longitudinalidade & 0,400 & 0,160 & 0,095 & 0,138 & 0,191 & $-0,56$ & 0,365 & 0,134 & 0,065 & 0,444 & 0,197 & 0,135 \\
\hline Coordenação & 0,557 & 0,310 & 0,257 & 0,673 & 0,453 & 0,411 & 0,752 & 0,565 & 0,531 & 0,559 & 0,313 & 0,260 \\
\hline Integralidade & 0,570 & 0,325 & 0,273 & 0,780 & 0,608 & 0,578 & 0,733 & 0,537 & 0,501 & 0,540 & 0,292 & 0,237 \\
\hline $\begin{array}{l}\text { Orientação } \\
\text { Familiar }\end{array}$ & 0,412 & 0,170 & 0,106 & 0,366 & 0,134 & 0,674 & 0,452 & 0,204 & 0,143 & 0,329 & 0,108 & 0,032 \\
\hline $\begin{array}{l}\text { Orientação } \\
\text { Comunitária }\end{array}$ & 0,636 & 0,405 & 0,359 & 0,572 & 0,327 & 0,275 & 0,610 & 0,372 & 0,324 & 0,467 & 0,218 & 0,158 \\
\hline $\begin{array}{l}\text { Competência } \\
\text { Cultural }\end{array}$ & 0,682 & 0,466 & 0,425 & 0,649 & 0,421 & 0,376 & 0,649 & 0,422 & 0,377 & 0,574 & 0,329 & 0,277 \\
\hline
\end{tabular}

Fonte: Elaboração própria. 
Na variável dependente inovação de processo, o maior percentual encontrado de variação foi explicado pela variável independente, integralidade $r^{2}=0,608(60,80 \%)$. Na variável dependente inovação de marketing, o maior coeficiente de determinação apresentou-se em $\mathrm{r}^{2}=0,565$, ou seja, indica que para cada unidade de mudança de coordenação se produziu uma unidade de mudança de 0,565 (56,50\%) na inovação de marketing. Por fim, na variável dependente organizacional, o maior percentual encontrado de variação foi explicado pela variável independente competência cultural $r^{2}=0,329$ (32,90\%), e para essa avaliação observa-se uma condição mais homogênea de participação das variáveis independentes sobre as dependentes.

O coeficiente de correlação ao quadrado ajustado ( $r^{2}$ ajustado), pela quantidade de variáveis independentes utilizadas, para fazer uma estimativa, avalia se houve ganho ou perda pela inclusão de uma nova variável no modelo, ou seja, se o valor apresentar-se diminuindo ou negativo, tem pouco poder explicativo, ou até mesmo representa uma explicação inversa ao modelo proposto (Hair Jr. et al., 2006). Considerando a tabela 3, ressalta-se a relação inversa da variável dependente inovação de processo em relação à variável independente dos escores parciais de acesso e a longitudinalidade (atendimento continuado), demonstrando que essas variáveis independentes possuem um valor explicativo inverso na variável dependente inovação de processo.

Observou-se também que, em relação à análise do coeficiente de correlação ao quadrado ajustado ( $r^{2}$ ajustado), temos a confirmação da análise do coeficiente de determinação $\left(\mathrm{r}^{2}\right)$, que indica que a variável independente de competência cultural tem o maior valor explicativo na variável dependente inovação de produto, sendo, portanto, relevante sua inclusão e participação nessa análise discriminada. Assim como a variável independente integralidade para a variável dependente inovação de processo, a variável independente coordenação para a variável dependente inovação de marketing e a variável independente competência cultural para a variável dependente inovação organizacional.

Visando o entendimento da contribuição de quanto as variáveis independentes contribuem para as variáveis dependentes, procurou-se avaliar o coeficiente angular de regressão (B), que determina a variação em unidade de uma variável dependente para cada variação da variável independente. Concomitantemente, procurou-se com a significância em $\mathrm{F}$ testar a significância da variável independente sobre uma variável dependente, de forma a comprovar o comportamento da associação e influência entre elas (Pestana e Gageiro, 2005).

A apresentação dos dados encontra-se na tabela 4, com destaque para os resultados significativos da correlação encontrada previamente.

Na variável dependente inovação de produto podemos visualizar que, se incrementarmos uma unidade da variável independente competência cultural, se produz um aumento de 0,312 unidade em inovação de produto $(F=0,005)$.

Na variável dependente inovação de processo, observamos que, se incrementarmos uma unidade da variável independente integralidade, se produz um aumento de 0,726 unidade em inovação de processo $(\mathrm{F}=0,001)$. 
Na variável dependente inovação de marketing, podemos visualizar que, se incrementarmos uma unidade na coordenação, se produz um aumento de 0,702 unidade em inovação de marketing $(\mathrm{F}=0,001)$.

Por fim, na variável dependente inovação organizacional, observamos que, se houver incremento da variável independente competência cultural, se produz um aumento de 0,286 unidade em inovação organizacional $(\mathrm{F}=0,025)$.

Ressalte-se que foram evidenciadas outras relações e influências significativas, porém, a fim de estabelecer uma análise conjunta da regressão linear simples, optou-se pela abordagem da influência de maior significância entre o cruzamento das 11 variáveis.

Tabela 4

Coeficiente de regressão e significância das variáveis da inovação e PCATool

\begin{tabular}{|c|c|c|c|c|c|c|c|c|}
\hline $\begin{array}{c}\text { Variável } \\
\text { Dependente }\end{array}$ & \multicolumn{2}{|c|}{ Produto } & \multicolumn{2}{|c|}{ Processo } & \multicolumn{2}{|c|}{ Marketing } & \multicolumn{2}{|c|}{ Organizacional } \\
\hline Variável & Coeficient & ignificância & Coeficiente & Significância| & Coeficiente & Significância & Coeficiente & Significância \\
\hline Independente & B & $\mathrm{F}$ & B & $\mathrm{F}$ & B & $\mathrm{F}$ & B & $\mathrm{F}$ \\
\hline Acesso & 0,230 & 0,900 & 0,099 & 0,481 & 0,199 & 0,151 & 0,185 & 0,222 \\
\hline Longitudinalidade & 0,228 & 0,140 & 0,078 & 0,623 & 0,210 & 0,180 & 0,277 & 0,098 \\
\hline Coordenação & 0,516 & 0,310 & 0,615 & 0,006 & 0,702 & 0,001 & 0,566 & 0,030 \\
\hline Integralidade & 0,538 & 0,270 & 0,726 & 0,001 & 0,697 & 0,002 & 0,556 & 0,380 \\
\hline $\begin{array}{c}\text { Orientação } \\
\text { Familiar }\end{array}$ & 0,239 & 0,127 & 0,209 & 0,180 & 0,264 & 0,091 & 0,208 & 0,232 \\
\hline $\begin{array}{l}\text { Orientação } \\
\text { Comunitária }\end{array}$ & 0,354 & 0,011 & 0,314 & 0,026 & 0,343 & 0,016 & 0,284 & 0,079 \\
\hline $\begin{array}{c}\text { Competência } \\
\text { Cultural }\end{array}$ & 0,312 & 0,005 & 0,293 & 0,009 & 0,299 & 0,009 & 0,286 & 0,025 \\
\hline
\end{tabular}

Fonte: Elaboração própria.

\section{Considerações finais}

A inovação é um fator importante para o desenvolvimento de um ambiente de mudanças, objetivando o aperfeiçoamento e a qualificação da oferta de produtos e serviços prestados para a sociedade. Observa-se que a meta fundamental da atenção primária é obter ganhos de saúde sustentáveis, mediante promoção, prevenção, tratamento e reabilitação, e, consequentemente, a condução de melhoria do bem-estar e, por conseguinte, o crescimento econômico e social. 
O objetivo geral do artigo foi identificar uma aproximação de elementos que qualificam um serviço de saúde orientado para a atenção primária, por meio do PCATool, e a tipologia de inovações evidenciadas. O estudo foi realizado no município de Caxias do Sul, pois se constitui como um referencial de serviços de saúde, na região nordeste do estado do Rio Grande do Sul, apresentando especial importância pelo comprometimento de esforços no aprimoramento dos serviços de saúde para a população desde 1997.

Na análise da correlação de Pearson das 15 UBS avaliadas foi possível constatar que, quanto maior o escore do PCATool no desenvolvimento da qualidade dos serviços prestados à população, decorrente dos atributos essenciais e derivados, maior o número de inovações encontradas. Essa relação permitiu mostrar que, de fato, existe uma evidência de associação, respeitando as características de cada UBS, no que tange a acessibilidade aos serviços, o atendimento continuado para os indivíduos, a oferta de produtos e serviços à população, a coordenação para outros níveis do sistema de saúde, a ESF, a intervenção de serviços de saúde junto à comunidade e a capacitação da equipe das UBS, com o número de inovações evidenciadas.

Assim, motivados pela análise da influência e relação específica da variável independente sobre uma variável dependente, foi demonstrado que, para as inovações de produto, existe uma influência significativa do atributo derivado competência cultural, do PCATool. Em outras palavras, o incremento de capacitação gerencial na organização das UBS e, também, na capacidade de oferecer serviços especiais à população adscrita estimula o aumento das inovações de produto. Embora exista relação com o atributo essencial da integralidade, que pressupõe a variedade de produtos e serviços disponíveis, é na competência cultural que se encontra a influência significativa para a introdução das inovações de produto.

No que tange à variável inovação de processo, foi possível constatar que a influência significativa acontece pelo atributo essencial da variável integralidade. Em outras palavras, o incremento na variedade de serviços disponíveis de maior igualdade, entre as UBS, com reconhecimento adequado dos problemas biológicos, psicológicos e sociais que causam as doenças, pressupõe um forte arranjo na organização e, consequentemente, ao estímulo no incremento das inovações de processo.

Ressalta-se a relação inversa, da variável inovação de processo, demonstrada pela análise do coeficiente de correlação ao quadrado ajustado, com a variável independente de acesso à UBS e o atendimento continuado ao longo dos anos. Ou seja, pressupõe-se que estimular a melhoria da variável acesso e longitudinalidade não acarretará o aumento das inovações de processo. Em outras palavras, o desenvolvimento de iniciativas que estendam o horário de atendimento à população, práticas que visem o acolhimento de todos os indivíduos, mudanças que permitam agilidade de atendimento ao usuário não pressupõem maior incremento de inovação de processo.

Em termos da variável inovação de marketing, observamos uma influência significativa com o atributo essencial da variável coordenação, ou seja, a capacidade de integração da equipe de trabalho com a população, associada a um pontual sistema de informação que conduza ao acompanhamento dos indivíduos para outros níveis de atenção à saúde, pressupõe um incremento nas inovações de marketing. 
Na variável inovação organizacional, observou-se a influência significativa com o atributo derivado da variável competência cultural. Em outras palavras, o incremento para a oferta de serviços especiais e o reconhecimento das necessidades das populações, devido a essas características especiais, que envolvem um arranjo organizacional diferenciado na prestação de serviço pela UBS, pressupõem um incremento nas inovações organizacionais.

Essas evidências permitem corroborar a dinâmica dos estudos da inovação no setor saúde, mostrando-se fundamentais para compreensão de políticas de promoção à saúde, e, consequentemente, do desenvolvimento local.

\section{Referências}

AHMED, Pervaiz K. Benchmarking innovation best practice: European Centre of Total Quality Management. Bradford: University of Bradford, 2001.

ALBUQUERQUE, Eduardo M.; CASSIOLATO José. As especificidades do sistema de inovação do setor saúde: uma resenha da literatura como introdução a uma discussão sobre o caso brasileiro. Belo Horizonte: Federação de Sociedades de Biologia Experimental, 2000. Estudos Fesbe. Disponível em: $<$ http://amxinformatica.com.br/redesist/images/projeto_saude/textos/documentos/material_bibliografico/Albuquerque_E._M._Cassiolato_J.E._As_especificidades_do_sistema.pdf $>$. Acesso em: 3 out. 2009.

ALVES-MAZZOTTI, Alda J.; GEWANDSZNAJDER, Fernando. O método nas ciências naturais e sociais: pesquisa qualitativa e quantitativa. 2. ed. São Paulo: Pioneira Thomson Learning, 1999.

BARDIN, Laurence. Análise de conteúdo. Lisboa: Edições 70, 2006.

BESSANT Jonh R.; TIDD, Joe. Innovation and entrepreneurship. Chichester: John Wiley \& Sons, 2007.

BODENHEIMER, Paul. Primary care - will it survive? New England Journal of Medicine, v. 43, n. 32, p. 56-72, 2006.

BRASIL. Ministério da Saúde. Secretaria de Atenção em Saúde. Departamento de Atenção Básica. Manual do instrumento de avaliação da atenção primária à saúde: primary care assessment tool pcatool - Brasil / Ministério da Saúde, Secretaria de Atenção em Saúde, Departamento de Atenção Básica. Brasília: Ministério da Saúde, 2010.

BRASIL. Ministério da Saúde. Sistema de Informações sobre Orçamentos Públicos em saúde. 2011. Disponível em: <http://siops.datasus.gov.br/municipio.caxiasdosul.php>. Acesso em: 12 maio 2012.

CAMPOS, Gastão W. S. Reforma da reforma: repensando a saúde. São Paulo: Hucitec, 1992.

CAXIAS DO SUL. Prefeitura Municipal de Caxias do Sul. Plano Municipal de Saúde. Secretaria do Planejamento Urbano. Plano Físico Urbano de Caxias do Sul. Caxias do Sul, 2004. 
CLAUS, Suzete M. Gestão de sistemas locais de saúde. Desafios cotidianos para o desenvolvimento de competências profissionais. Caxias do Sul: Educs, 2007.

CLAUS, Suzete M.; CAPRA, Margareth . Potencializando a gestão para organizar o SUS a partir da Rede Básica. Porto Alegre: Dacasa, 2002.

CONASS. Conselho Nacional de Secretários de Saúde. Atenção Primária e promoção da saúde. Brasília: Coleção Progestores/Para entender a Gestão do SUS, 2007.

COOKE, Paul; MORGAN, Karl. The associational economy: firms, regions and innovation. Nova York: Oxford University Press, 1998.

DAMANPOUR, Fariborz; SZABAT, Paul; EVAN, Christian. The relationship between types of innovation and organizational performance. Journal of Managment Studies, v. 26, n. 6, p. 45-98, 1989.

DEN EXTER, André el at. Health care systems in transition: Netherlands. Copenhague: WHO Regional Office for Europe on Behalf of the European Observatory on Health Systems and Policies, 2004.

DOSI, Giovanni. The nature of the innovative process. In: DOSI, Giovanni et al. Technical change and economic theory. Londres: Pinter, 1982. p. 94-113.

DRUCKER, Peter F. Fator humano e desempenho: o melhor de Peter Drucker sobre administração. São Paulo: Pioneira, 1981.

FLICK, Uwe. Uma introdução à pesquisa qualitativa. 2. ed. Porto Alegre: Bookman, 2004.

GADELHA, Carlos A. et al. Inovação em saúde: dilemas e desafios de uma instituição pública. Rio de Janeiro: Fiocruz, 2007.

GAULD, Robin et al. The World Health report 2008 - Primary healthcare: how wide is the gap between its agenda and implementation in 12 high-income health systems? Healthcare Policy, v. 7 , n. 3, p. 38-58, 2012.

GOFIN, Jaime; GOFIN, Rosa; CUETO, Marcos. Community-oriented primary care and primary health care. American Journal Public Health, v. 95, n. 5, p. 757, 2005.

HAIR JR., Joseph F. et al. Fundamentos de métodos de pesquisa em administração. Porto Alegre: Bookman, 2006.

HARZHEIM, Erno; DUNCAN, Bruce; STEIN, Aírton. Quality and effectiveness of different approaches to primary care delivery in Brazil. BMC Health Services Research, Londres, v. 6, n. 156, p. 1-13, 2006.

HARZHEIM, Erno et al. Internal consistency and reliability of Primary Care Assessment Tool (PCATool-Brasil) for child health services. Cadernos de Saúde Pública, Rio de Janeiro, v. 22, n. 8, p. 1649-1659, 2006.

HEALTH EVIDENCE NETWORK. What are the advantages and disadvantages of reestructuring a health care system to be more focused on primary care services? Copenhague: World Health Organization, 2004. 
HESSELBEIN, Frances. Leading for innovation. San Francisco: Jossey-Bass, 2002.

HIGGINS, James M. Innovate or evaporate: test \& improve your organization's IQ: its innovation quotient. Ohio: New Management, 1995.

IBGE. Instituto Brasileiro de Geografia e Estatística. Disponível em: <www.ibge.gov.br/cidadesat/ painel/painel.php?codmun=430510>. Acesso em: 12 abr. 2013.

LEITE, Luiz F. Inovação: o combustível do futuro. Rio de Janeiro: Qualitymark, 2005.

MACINKO, James; ALMEIDA, Célia. Organization and delivery of primary health care services in Petropolis, Brazil. International Journal of Health Planning and Management, v. 4, n. 19, p. 303317, 2004.

MALERBA, Franco. Sectoral systems of innovation and production: concepts, analytical framework and empirical evidence. 1999. Disponível em: <www.druid.dk/uploads/tx_picturedb/ds199969.pdf>. Acesso em: 10 mar. 2011.

MANUAL DE OSLO. The measurement of scientific and technological activities. 2005.

MENDES, Eugenio V. Redes de Atenção à Saúde. Belo Horizonte: Secretaria de Estado de Saúde de Minas Gerais, 2007.

MIEDZINSKI, Maurice. Development policy as a regional innovation policy? Local innovation and knowledge to enhance capacity development. The young think tank of the Club of Rome. 2006. Disponível em: <www.clubofrome.org/tt30/material/knowledge_sharing_michal.pdf>. Acesso em: 10 dez. 2010.

NODARI, Cristine H. et al. Innovations in primary care management: a Brazilian experience. World Review of Entrepreneurship, Management and Sustainable Development, v. 8, n. 2, p. 165-180, 2012.

PEREIRA, João Pedro C. N.; CARVALHO, Marly M.; LAURINDO, Fernando B. Estrutura do arranjo produtivo, da governança e competência coletiva como fatores da competitividade local: um estudo exploratório no município de Holambra-SP. 2003. Disponível em: <www.simpep.feb.unesp.com. br/Artigos\%20Apresentados.htm>. Acesso em: 9 dez. 2009.

PESTANA, Maria H.; GAGEIRO, João N. Análise de dados para ciências sociais: a complementariedade do SPSS. 4. ed. rev. e aum. Lisboa: Sílabo, 2005.

POTTS, John; KASTELLE, Tina. Public sector innovation research: what's next? Innovation: Management. Policy \& Practice, v. 12, n. 2, p. 122-137, 2010.

ROTHWELL, Richard. Industrial innovation: success, strategy, trends. In: DODGSON, Mark; ROTHWELL, Richard. The handbook of industrial innovation. Cheltenham: Edward Elgar, 1996.

SCHUMPETER, Joseph A. The theory of economic development. Cambridge: Harvard University Press, 1934.

SECRETARIA DO DESENVOLVIMENTO ECONÔMICO. Prefeitura Municipal de Caxias do Sul. 2008. Disponível em: <www.caxias.rs.gov.br/>. Acesso em: 10 jan. 2011. 
STARFIELD, Barbara. Atenção primária: equilíbrio entre necessidades de saúde, serviços e tecnologia. Brasília: Unesco; Ministério da Saúde, 2002.

STARFIELD, Barbara. Is primary care essential? Lancet, v. 344, n. 8930, p. 1129-1133, 1994.

STARFIELD, Barbara; SHI, Leiyu; XU, Jiahong. Validating the adult primary care assessment tool. Journal of Family Practice, v. 50, n. 2, p. 161-175, 2001.

TIDD, Joe; BESSANT, Jonh R.; PAVITT, Keith. Managing innovation: integrating technological, market and organizational change. 3. ed. Chichester: John Wiley \& Sons, 2005.

TIGRE, Paulo B. Gestão da inovação. A economia da tecnologia no Brasil. Rio de Janeiro: Elsevier, 2006.

TOIVONEN, Marja; TUOMINEN, Tiina. Emergence of innovations in services. Service Industries Journal, v. 29, n. 7, p. 887-902, 2009.

VEN, Arold H. V. Research on the management of innovation: the Minnesota studies. Nova York: Oxford University Press, 1999.

VUORI, Hannu V. Primary care: is there enough time for prevention? American Journal of Public Health, v. 93, n. 41, p. 635-641, abr. 1992.

WORLD BANK. World development report 1993: Investing in Health. Oxford: Oxford University, 1993.

WORLD HEALTH ORGANIZATION. The world health report 2000: health systems, improving performance. Geneva: WHO, 2000.

WORLD HEALTH ORGANIZATION. Towards a conceptual framework for analysis and action on the social determinants of health. Geneva: WHO, 2005. (Discussion Paper, n. 5)

YIN, Robert K. Estudo de caso: planejamento e método. 2. ed. Porto Alegre: Bookmann, 2005.

Cristine Hermann Nodari é doutoranda do Programa de Pós-Graduação em Administração da Universidade de Caxias do Sul (PPGA-UCS). E-mail: cristine.nodari@gmail.com.

Pelayo Munhoz Olea é doutor em administração e direção de empresas pela Universitat Politècnica de Catalunya e professor do Programa de Pós-Graduação em Administração da Universidade de Caxias do Sul (PPGA-UCS).E-mail: pelayo.olea@gmail.com.

Eric Charles Henri Dorion é doutor em administração pela Université de Sherbrooke e professor do Programa de Pós-Graduação em Administração da Universidade de Caxias do Sul (PPGA-UCS). E-mail: echdorion@gmail.com. 